\title{
Perspective
}

PERSPECTIVE Actualité en histoire de l'art

$4 \mid 2009$

$\mathrm{XX} /\left.\mathrm{XX}\right|^{\mathrm{e}}$ siècles

\section{Les éditions d'artistes depuis les années 1960 : livres, revues et multiples}

Artists' editions since the 1960s: books, journals and multiples

\section{Laurence Corbel}

\section{(2) OpenEdition}

1 Journals

\section{Édition électronique}

URL : http://journals.openedition.org/perspective/1280

DOI : $10.4000 /$ perspective. 1280

ISSN : 2269-7721

Éditeur

Institut national d'histoire de l'art

\section{Édition imprimée}

Date de publication : 31 décembre 2009

Pagination : 581-588

ISSN : 1777-7852

\section{Référence électronique}

Laurence Corbel, «Les éditions d'artistes depuis les années 1960 : livres, revues et multiples »,

Perspective [En ligne], 4 | 2009, mis en ligne le 07 août 2014, consulté le 01 octobre 2020. URL : http:// journals.openedition.org/perspective/1280; DOI : https://doi.org/10.4000/perspective.1280 


\section{Les éditions d'artistes depuis les années 1960 : livres, revues et multiples}

\section{Laurence Corbel}

- Leszek Brogowski, Anne Meglin-Delcroix éd., Livres d'artistes : l'esprit de réseau, (colloque, Rennes, 2003), numéro spécial de la Nouvelle Revue d'Esthétique, 2, octobre 2008. 160 p., fig. en n. et b. ISBN : 978-2-13057019-6;30€.

- Ulises CARrión, Quant aux livres, On Books, Juan J. Agius éd., Genève, Éditions Héros-Limite, (1997) 2008, édition bilingue. 210 p., ISBN : 978-2-970030-01-0; $20 €$.

- Eye on Europe: Prints, Books, e Multiples, 1960 to Now, Deborah Wye, Wendy Weitman éd., (cat. expo., New York, The Museum of Modern Art, 2006-2007), New York, MoMA, 2006. 324 p., 354 fig. en coul., ISBN : 978-0-87070-371-3; \$65 (48€).

- Yves Jolivet éd., Le livre et l'artiste, (colloque, Marseille, 2007), Marseille, Le mot et le reste, 2007. 224 p., fig. en n. et b. ISBN : 978-2-9153-7847-4; $19 €$.

- Anne Mogglin-Delcroix éd., Sur le livre d'artiste : articles et écrits de circonstance (1981-2005), Marseille, Le mot et le reste, (2006) 2008. 588 p., fig. en n. et b. ISBN : 2-915378-29-0;29€.

- Revues d'artistes. Une sélection, Marie Boivent éd., (cat. expo., Fougères, Galerie des Urbanistes/Rennes, Lendroit Galerie, Cabinet du livre d'artiste, 2008), Fougères, Association Arcade/Paris, Éditions Provisoires/Rennes, Lendroit Galerie, 2008. 264 p., 110 fig. en n. et b. ISBN : 978-2-9517-1047-4; $15 €$.

Les années 1960, qui constituent un tournant dans l'histoire de l'art contemporain, ont vu se développer de nouvelles pratiques et formes artistiques. Parmi celles-ci les multiples, ces œuvres destinées à être produites en plusieurs exemplaires, occupent une place de choix tant par la diversité des techniques et des médiums qu'ils utilisent que par les changements qu'ils introduisent du point de vue de la diffusion et de la réception de l'art ${ }^{1}$. Cette catégorie de multiple, qui répond au processus de dé-spécification de l'art en vigueur à cette période, permet de rassembler et de nommer des objets et des imprimés inclassables si l'on s'en tient aux cadres et typologies traditionnels de l'histoire de l'art.

Avec le multiple, il ne s'agit jamais seulement pour les artistes de mettre les possibilités offertes par de nouvelles techniques au service de l'art ; leur projet est aussi d'enrichir les pratiques artistiques et de permettre une diffusion élargie des œuvres. Le multiple participe donc d'une redéfinition de l'art à moins que ce ne soit, pour reprendre l'expression d'Harold Rosenberg, de sa "dé-définition ${ }^{2}$, de la transgression des frontières artistiques traditionnelles, du rapprochement de l'art et de la vie - autant de traits caractéristiques des changements qui affectent l'art des années 1960 -, et de la mise en place de nouveaux circuits de diffusion, en marge voire en opposition aux circuits muséaux et marchands traditionnels. Pour Anne Mœglin-Delcroix, spécialiste du livre d'artiste en France, l'appellation même de "livre d'artiste " implique en outre "une redéfinition en vérité très précise de la fonction artistique et une mutation historique du statut de l'artiste : sur fond d'une crise des disciplines artistiques traditionnelles [...] se développe une conception quasi expérimentale de la création, qui [...] procède par appropriation artistique de moyens non-artistiques " (MEgLIN-DelCroiX, 2008, p. 82).

Si les formes artistiques émergentes des années 1960 telles que l'art de la performance ou de la vidéo ont, dès leur apparition, fait l'objet de nombreuses études, il a fallu attendre presque deux décennies pour que l'art du multiple, notamment les publications d'artistes, suscite l'intérêt des chercheurs. Un tel retard peut s'expliquer par leur diffusion qui, passant souvent par des réseaux parallèles et confidentiels, a pu constituer un obstacle à leur appropriation, mais il est aussi certainement imputable à leur caractère ordinaire et souvent peu attractif. Plusieurs ouvrages récents mettent à la portée d'un public élargi cet aspect de l'histoire de l'art contemporain demeuré jusqu'ici dans le cercle restreint de spécialistes ou d'amateurs, mais dont la complexité et la richesse soulèvent de nombreuses questions et suscitent des dialogues qui engagent autant la théorie de l'art contemporain que sa pratique, voire sa définition.

\section{Multiples multiples}

Le catalogue publié à l'occasion de l'exposition Eye on Europe: Prints, Books, e Multiples, 1960 to Now, organisée au MoMA entre octobre 2006 
et janvier 2007, dresse un large panorama de la manière dont les artistes ont développé la pratique du multiple depuis les années 1960 (Eye on Europe..., 2006). Les commissaires, Deborah Wye et Wendy Weitman, se proposent de faire une synthèse de l'évolution de l'art européen à travers les médiums de l'imprimé, du livre et des multiples, et de mettre en lumière la manière dont ils participent à la constitution de l'esthétique contemporaine. Ce projet, qui revendique la spécificité d'un point de vue américain et même new yorkais sur le sujet, pèche par sa trop grande ambition, qui le conduit à rassembler des ouvres emblématiques d'une "nouvelle sensibilité " (p. 16) mais dont l'esprit diffère parfois radicalement lorsqu'on les examine de près. En effet, le parti pris de cette exposition tend à réduire l'hétérogénéité de cette production. Qu'y a-t-il de commun entre l'œuvre gravée sur linoleum à exemplaire unique de Georg Baselitz (p. 158), la sérigraphie de Gerhard Richter éditée à vingt exemplaires (p. 56), le livre d'artiste Sans-Souci de Christian Boltanski publié en 1991 en deux mille exemplaires (p. 98) et les Optimistic Boxes de Robert Filliou en édition illimitée (p. 125 ; fig. 1) ? Excepté leur appartenance à la catégorie de multiples,

1a. Ben Vautier : Moi, Ben je signe, 1975, New York, MoMA ; Théâtre d'art total, 1964 New York,

MoMA ; Art does not exist, 1989 ; Take your time, 1998, New York, MoMA Library ; b. Robert Filliou, The Frozen Exhibition, 1972 Brême, Neues Museum Weserburg ; Optimistic Boxes 1-5, 19681981, New York, MoMA [Eye on Europe..., 2007, p. 124-125].

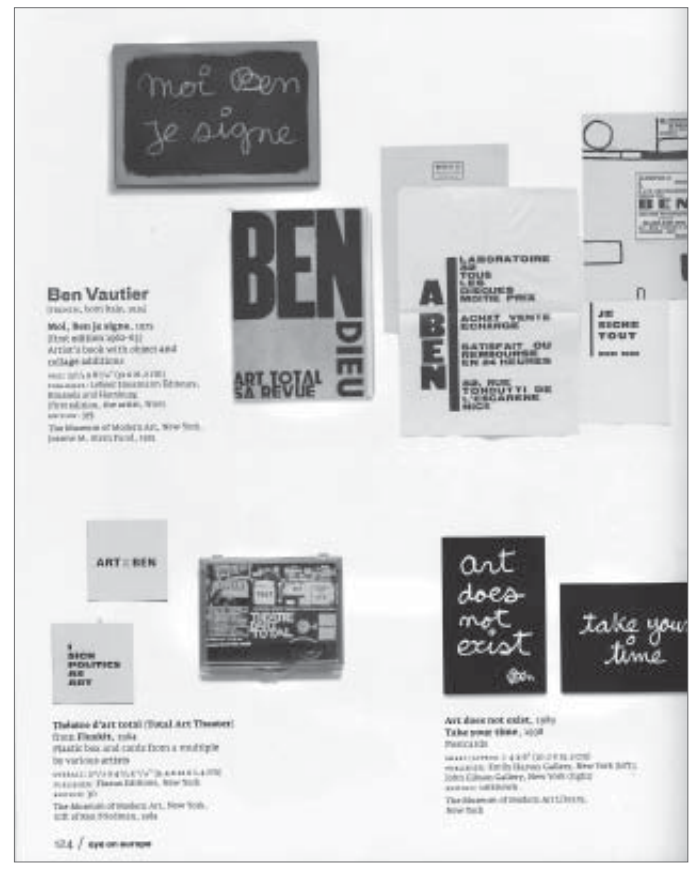

ces travaux ont en réalité un statut très différent : les œuvres numérotées et signées, au tirage limité, qui prennent place sur les cimaises des galeries ou des musées sont plus proches du format traditionnel de l'œuvre d'art que de celui des multiples, au tirage illimité, qui ont radicalement transformé les conditions de diffusion et de réception de l'art.

À la décharge des commissaires, il faut rappeler que la définition du terme " multiple " est au cœur de nombreuses discussions ${ }^{3}$. De fait, le catalogue, qui rassemble les travaux de cent dix artistes et collectifs issus de vingt pays, propose des reproductions, toutes en couleurs et de qualité, qui donnent à voir la diversité des multiples tant dans les supports - livres, journaux, revues, cartes postales, objets, stickers, papiers peints, flyers, affiches et éphémeras - que dans les techniques, notamment dans les techniques d'impression utilisées - gravure, lithographie, sérigraphie, offset, photocopie. Mais ce nivellement est peut-être également un effet de l'exposition qui transforme le statut particulier de certains de ces multiples que sont les livres et les revues d'artistes : d'objets à lire, à manipuler, à feuilleter, ils sont réduits, une fois placés sous vitrines, au statut d'objets à voir.

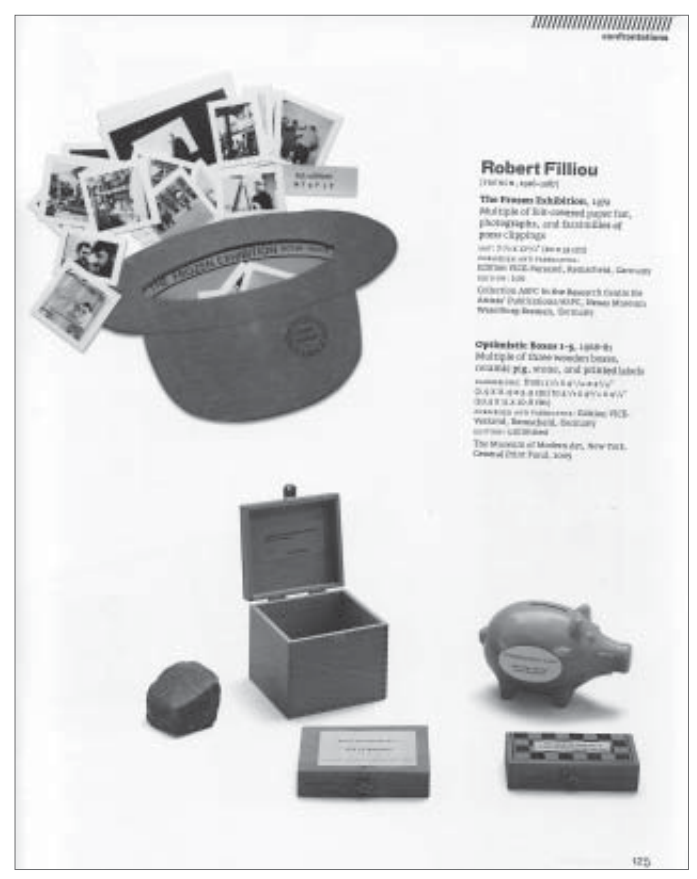




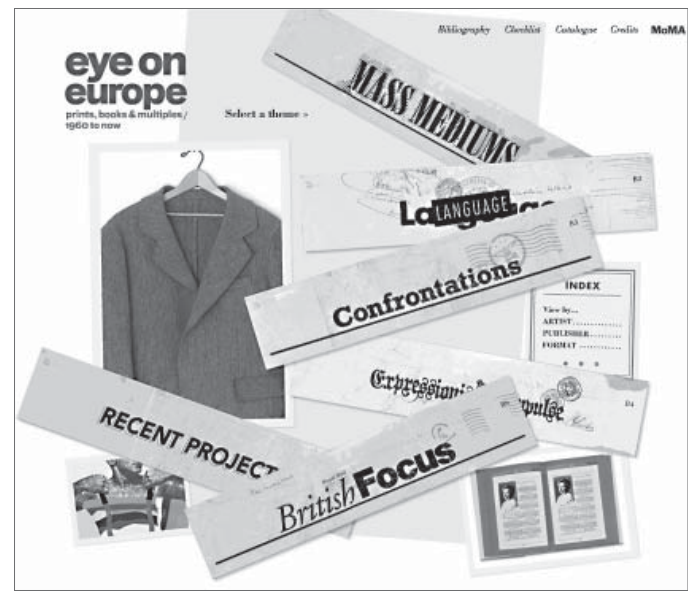

L'introduction au catalogue du MoMA présente les six thématiques de l'exposition (fig. 2) ${ }^{4}$, élaborées selon des critères disparates - "mass médiums ", " langage ", " confrontations ", " élan expressionniste ", " projets récents ", "focus britannique " - et propose également, précédée d'une mise en perspective historique, une analyse intéressante du " monde de l'imprimé " de la seconde moitié du XXe siècle en Europe, notamment du réseau que constituent les lieux d'archivage, les institutions muséales, les librairies, les revues spécialisées et les événements organisés autour de la diffusion de ces pratiques artistiques. Ces réseaux, essentiellement institutionnels et donc dotés de visibilité, diffèrent de ceux plus confidentiels qui échappent aux circuits habituels de l'art. Il est incontestable, comme le disent Deborah Wye et Wendy Weitman, que l'apparition de nouvelles techniques a été un facteur déterminant pour l'émergence et le développement de ces formes artistiques multiples, mais on sait que la rupture ne tient pas tant aux techniques utilisées qu'à l'idée que l'on se fait de l'art et à la volonté de transformer le statut social, politique et économique de celui-ci : elle est avant tout le fait d'artistes qui veulent créer un espace d'expérimentation étranger à toute spéculation commerciale. De ce point de vue, les publications d'artistes ont, parmi les multiples, un statut spécifique en ce qu'elles ont contribué à modifier les manières de faire de l'art et de le diffuser, en contournant le pouvoir des institutions et en luttant contre la marchandisation de l'art.
Les publications d'artistes : un objet de recherche en plein essor

Spécifiquement consacrées aux multiples des $\mathrm{XX}^{\mathrm{e}}$ et $\mathrm{XXI}^{\mathrm{e}}$ siècles et aux formes particulières de cet art que sont les livres et les revues d'artistes, les publications recensées ici témoignent du dynamisme des recherches et de la volonté de les diffuser au-delà du cercle restreint des collectionneurs, des spécialistes et des acteurs auquel elles restent trop souvent cantonnées. Après avoir été longtemps une terra incognita, le livre d'artiste est aujourd'hui un terrain privilégié de recherche en art contemporain.

Un des traits remarquables de ce champ est ainsi le décloisonnement entre théorie et pratique. Les chercheurs qui travaillent sur le sujet sont aussi engagés dans l'édition de livres et de revues d'artistes ou dans le commissariat d'exposition, contribuant en effet à sa diffusion. Les travaux de Mœglin-Delcroix témoignent de cette dimension militante qui s'exprime dans la défense $\mathrm{d}^{\prime}$ " une certaine idée du livre d'artiste " (MogGLIN-DelCRoix, 2008, p. 23) et dans l'organisation de plusieurs expositions dont elle a été la commissaire. Leszek Brogowski, qui dirige une équipe d'accueil à l'université Rennes 2, a fondé en 2000 les éditions Incertain Sens qui développent, avec la publication de livres d'artistes, un programme de recherche autour des questions telles que la fonction critique de l'art, le statut de l'œuvre, la sociologie des échanges, le nouveau rapport du public à l'œuvre, et se proposent de contribuer à la construction d'un réseau d'échanges ou d'information et de coopération internationale ${ }^{5}$. Dans sa communication au colloque de Marseille, "Voir le livre, voir le jour. Comment j'ai fabriqué et lu certains de 'mes' livres ", Brogowski analyse cette expérience éditoriale comme un "paradigme du travail de recherche " qu'il mène au sujet de l'art (Jolivet, 2007, p. 154), et souligne le lien étroit entre cet engagement éditorial et son activité d'enseignant et de chercheur. Marie Boivent, dont les recherches portent sur la revue d'artiste depuis les années 1960, est elle aussi éditrice de publications d'artistes ${ }^{6}$ et commissaire de l'exposition Revues d'artistes.
2. Page d'accueil du site Internet de l'exposition Eye on Europe..., MoMa, New York, 2006-2007 (www.moma. org/interactives/ exhibitions/2006/ eyeoneurope/ flash.html). 
Une sélection organisée en 2008. Parallèlement, les artistes qui ont fait du livre un médium artistique sont souvent des théoriciens de cette pratique. Quant aux livres. On Books, dont une réédition vient de paraître, rassemble des textes publiés dans les années 1970 d'Ulises Carrión, figure exemplaire de cette articulation de la théorie et de la pratique : artiste, auteur, éditeur de livres d'artistes, libraire et théoricien, Carrión apporte avec ses textes une contribution décisive pour la théorie du livre d'artiste (CARRIón, [1997] 2008).

Composée de préfaces de catalogues d'exposition ou de catalogues raisonnés et d'articles publiés dans des revues ou des ouvrages collectifs, l'anthologie de Mœeglin-Delcroix, Sur le livre d'artiste. Articles et écrits de circonstance (1981-2005), présente des analyses qui prolongent et infléchissent la réflexion qu'elle développa il y a dix ans dans Esthétique du livre $d^{\prime}$ artiste $^{7}$. Tout en réaffirmant ses thèses principales, l'auteur, qui précise dans une courte présentation le contexte et les circonstances de la rédaction de chacun des textes, revient sur quelques-unes des analyses proposées dans son livre précédent pour apporter des nuances. Elle rectifie par exemple « une interprétation souvent trop conceptuelle, abstraite, mallarméenne du recours au livre" (MEGLIN-DELCROIX, 2008, p. 346), et prend en compte la pluralité de ses manifestations repérable dès le début des années 1960. Alors qu'Esthétique du livre d'artiste traitait principalement de la naissance et du développement du livre d'artiste en relation avec les avantgardes des années 1960 et 1970 en se concentrant sur les pionniers de cette pratique artistique, cette anthologie montre son évolution : certains articles analysent les récentes orientations de la pratique artistique d'une nouvelle génération d'artistes qui utilise le livre comme médium (Claude Closky, Marie-Ange Guilleminot, Pascal Le Coq, Roberto Martinez, Eric Watier), tandis que d'autres essais monographiques traitent d'un artiste en particulier (Maurizio Nannucci, Ian Hamilton Finlay, David Tremlett, etc.), attestant la diversité et l'inventivité des usages de ce support traditionnel qu'est le livre.
Le livre d'artiste : une identité controversée Une question soulevée par plusieurs des articles publiés dans ces ouvrages est celle des caractéristiques propres aux publications d'artiste : artistes et théoriciens s'attachent ainsi à faire ressortir la spécificité artistique de cette forme d'expression qui est sans commune mesure avec les œuvres d'art traditionnelles du fait de l'apparence souvent banale et du coût modique de cette production. Le livre et l'artiste, publication des actes du colloque organisé à Marseille en mai 2007 et auquel participaient des spécialistes issus d'horizons divers (artistes, éditeurs, responsables de collections spécialisées, universitaires historiens et philosophes de l'art), témoigne non seulement de l'extrême diversité de ce qui est désormais un genre artistique, mais aussi des divergences qui règnent autour de la conception du livre d'artiste et de sa définition. Cet ouvrage met notamment en relief l'opposition entre une approche inscrite dans la continuité de la tradition de fabrication artisanale du livre illustré ou du livre de peintre, présentée dans l'article « Le livre comme creuset " d'Yves Peyré (Jolivet, 2007, p. 33-68), et une conception qui définit le livre d'artiste, au contraire, comme un imprimé ordinaire. Loin de se réduire à une simple querelle de mots, cette question est centrale aussi bien d'un point de vue théorique que pratique et les enjeux qui sous-tendent ces divergences sont essentiels au regard de la conception que l'on se fait de l'art.

Le problème de sa définition constitue ainsi une véritable pierre d'achoppement pour ceux qui ont fait du livre d'artiste leur objet d'étude. Comme le remarque Mœglin-Delcroix, "les étiquettes ne sont pas innocentes et, partant, la question terminologique nullement secondaire " (MøgLIN-DELCROIX, 2008, p. 81). Si la définition du livre d'artiste est abordée par quasiment tous les théoriciens, c'est parce qu'elle recouvre des enjeux essentiels relatifs à son statut dans le monde de l'art. L'identité du livre d'artiste doit s'imposer contre une tendance répandue à le confondre avec le livre d'art et le livre de bibliophilie. Bien qu'utilisant le même médium que ceux-ci, le livre d'artiste s'en distingue, et s'y oppose même par ses visées politiques, comme l'explique Kate 


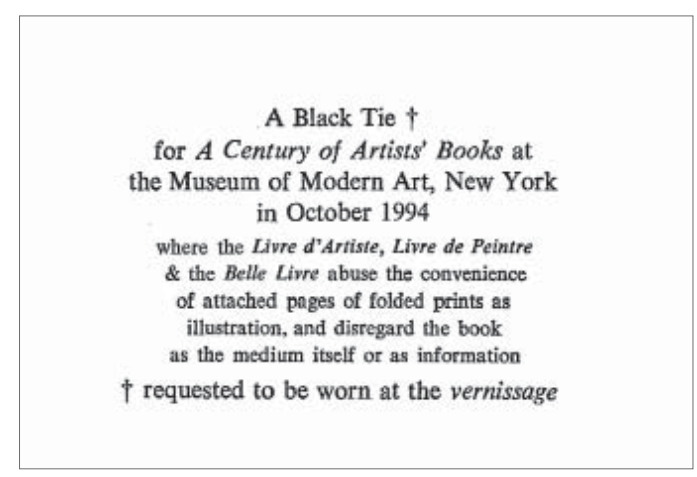

Linker dès 1980 dans «The Artist's Book as an Alternative Space ${ }^{8}$. En effet, le livre d'artiste $\mathrm{s}^{\prime}$ est revendiqué comme une pratique artistique alternative aux modes de diffusion traditionnels de l'art et résistante à sa marchandisation : c'est la récupération institutionnelle et marchande du livre d'artiste qui est ici en jeu. Lutter contre la dilution de la spécificité du livre d'artiste qui est un des symptômes de sa récupération dans les circuits institutionnels de l'art et contre un retour vers une conception plus traditionnelle de l'appropriation du livre par l'art, c'est essayer d'en sauvegarder la force critique. On comprend ainsi que les artistes ne sont pas en reste dans ces débats, comme le montre une des cartes postales réalisées par le fondateur de Coracle Press, Simon Cutts (p. 26 ; fig. 3), qui invite avec ironie à porter le deuil lors du vernissage de l'exposition A Century of Artists' Books, présentée au MoMA en $1994^{9}$, pour protester contre la confusion entre les livres illustrés ou livres de peintres, et les livres d'artistes. Paradoxalement, le succès de l'expression aura contribué à accroître les confusions en élargissant le livre d'artiste à toute espèce de livre où un artiste est intervenu ; d'où la nécessité d'établir des critères qui permettent de distinguer le livre d'artiste d'autres usages du livre dans le domaine artistique (voir CARRIÓN, p. 31-53) ${ }^{10}$.

Il reste qu'en dépit de ces efforts, l'identité du livre d'artiste demeure indécise, comme le montre le bref article de Clive Phillpot, « Booktrek: The Next Frontier " (1990) ${ }^{11}$. Tout en les distinguant des livres d'artistes de facture artisanale, l'auteur souligne le caractère indiscernable de ce qu'il nomme une "littérature grise ", signifiant ainsi que ces livres relèvent autant du genre " art " que du genre "livre" (Livres d'artistes...,
2008, p. 20). Phillpot remarque à ce propos que l'assimilation de ces livres à de l'art constitue un frein à l'élargissement à de nouveaux publics et qu'il serait opportun de les annexer à la production littéraire alternative pour qu'elles réalisent vraiment « leur potentiel de forme d'art 'démocratique' " (p. 19). Au titre des priorités de ces pratiques artistiques se trouve, en effet, le projet d'une diffusion élargie de l'art.

\section{Le réseau : une dimension essentielle des publications d'artistes}

S'il se situe en marge du monde de l'art, le livre d'artiste ne se revendique pas pour autant comme une pratique en retrait de la sphère sociale. Le numéro 2 de la Nouvelle Revue d'Esthétique, qui traite des liens existant entre le livre d'artiste et le réseau, montre à travers des articles et des documents d'artistes ou de théoriciens que cette insertion de l'art dans le tissu social est au cœur de cette pratique artistique, comme l'illustre le livre d'artiste, Art as Social Environnment, de Maurizio Nannucci (fig. 4) ${ }^{12}$. En effet, ce terme de "réseau " renvoie non pas "à une infrastructure technique mais à un esprit fonctionnant sur le mode de l'affinité qui cimente une communauté géographiquement sans frontière mais numériquement restreinte d'artistes, d'éditeurs, de lecteurs, de bibliothécaires, de critiques, de collectionneurs qui sont parfois les mêmes " (Livres d'artistes..., 2008, p. 7). Ainsi se constituent, dans les espaces laissés vacants par l'institution des réseaux dont Brogowski précise qu'ils " n'ont ni centre, ni limites" (JoliveT, 2007, p. 169). Le texte en forme de manifeste "Art in Bookform/Book in Artform " de Nannucci ${ }^{13}$,

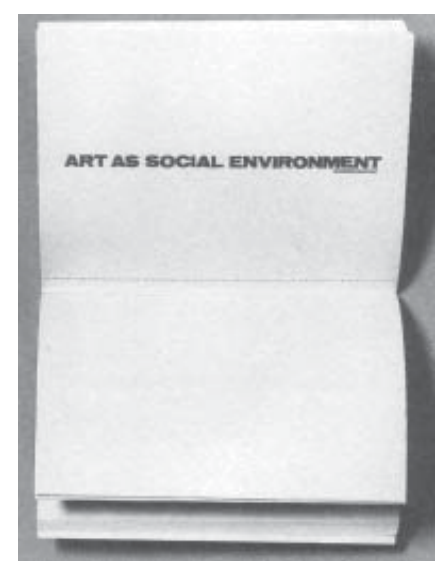

artiste italien qui défend l'idée d'un art multipliable et portable, sortant de l'espace des musées et des galeries, d'un art qui soit à la fois nulle part et partout, est une illustration de cet esprit de
3. Simon Cutts, A Black Tie, carte postale, 1995.
4. Maurizio Nannucci, Art as Social Environnment, Lugo, Exit/Amsterdam, Others Books and So, 1978 , 250 exemplaires. 
réseau qui a pour principe de s'épanouir sans limites. La critique d'art américaine Kate Linker montre, quant à elle, que le livre d'artiste représente "un espace alternatif absolu " qui fonctionne comme un espace d'exposition, un " espace d'art au-delà de l'espace ", grâce notamment à la pratique de l'autoédition et à la mise en place de nouveaux circuits de diffusion : le livre d'artiste opère, selon elle, un double glissement de l'objet vers l'information, de la galerie vers le texte $^{14}$. La pratique de l'œuvre multiple modifie ainsi les conditions d'accessibilité à l'art : elle élargit le cercle de ses spectateurs, devenus aussi des lecteurs, transforme la nature de l'expérience esthétique (la rencontre avec les œuvres n'a plus seulement lieu dans les espaces traditionnels de l'art que sont le musée et la galerie), et substitue souvent à l'acte créateur solitaire une entreprise collective impliquant plusieurs types de collaboration entre le ou les artistes, l'éditeur, l'imprimeur, le diffuseur, etc.

C'est aussi le format de l'exposition que les publications mettent en question, comme le remarque Marie Boivent, commissaire en 2008 de la première exposition en France de cette ampleur consacrée aux revues d'artistes. Elle ne manque pas de souligner dans le catalogue le paradoxe qu'il y a à exposer des revues d'artistes "qui sont mises hors circuit par le principe même de l'exposition " (Revues d'artistes..., 2008, p. 19). Brogowski formulait ce même constat - que l'on pourrait d'ailleurs étendre aux revues d'artistes - de façon plus radicale un an auparavant, affirmant que le livre d'artiste se prête mal à la présentation muséographique : "Un livre que l'on ne peut feuilleter n'est pas un livre. Lorsque le livre d'artiste est exposé sous une vitrine comme objet de collection, et à ce titre précieux et intangible, alors peu importe qu'il soit ouvert ou fermé, car, immobilisé sous le verre, il cesse d'être livre et devient son cadavre : un corps sans âme "

5. Pascal Le Coq, 4 de couverture montrant la courbe mensuelle des abonnés OXO au 31 décembre 2004, dans $O X O$, 30, 2004.
(JOLIVET, 2007, p. 184). Il y a là une tension certainement indépassable entre les critères institués de l'exposition et les exigences d'accessibilité d'une pratique artistique, tension qui invite à réfléchir à des modalités de présentation plus attentives à la spécificité des livres et des revues d'artistes. Ainsi, c'est la bibliothèque que Brogowski choisit comme alternative à la collection muséale.

Les revues d'artistes sont, plus encore que les livres d'artistes, confrontées à la question du réseau par le biais de la diffusion, qui constitue un de leurs éléments déterminants : de même que les livres d'artistes, elles pratiquent souvent, en marge des circuits éditoriaux traditionnels, l'autoédition et l'autodiffusion. Comme l'explique Boivent, "du don à l'achat, du hasard d'une 'rencontre' à l'attente d'une revue à laquelle on est abonné, la démarche de l'artiste et les conditions de réception du lecteur ne peuvent être les mêmes " (BROGOWSKI, Moglin-Delcroix, 2008, p. 57). Le catalogue d'exposition Revues d'artistes. Une sélection dirigé par Boivent donne un aperçu, fort bien documenté par des notices ou des entretiens avec les artistes, de l'éventail de pratiques en vigueur dans ce domaine. Cette sélection, qui rend compte de la diversité de ces revues - que de nombreux mouvements artistiques, de Fluxus à l'art conceptuel en passant par la poésie concrète, ont investi selon des périodicités et des durées variables - montre des pratiques artistiques animées par le désir de travailler collectivement et même de considérer les lecteurs comme un paramètre dans la réalisation de la

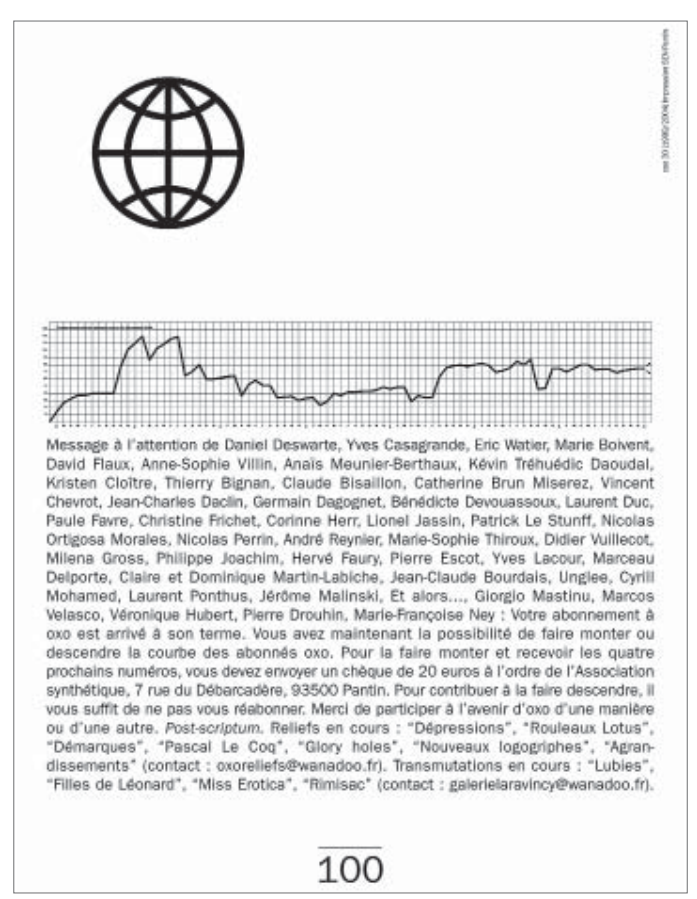


revue. Ainsi, la revue $O X O$ créée par Pascal Le Coq (fig. 5), à géométrie variable, voit-elle sa forme - pagination, technique, tirage - se rétracter ou se développer au gré du nombre de ses abonnés ${ }^{15}$. Ce principe, qui réévalue la place du lecteur, permet d'expérimenter un mode de production indépendant " libéré des contraintes financières et relationnelles inhérentes au marché de l'art " (Revues d'artistes..., 2008, p. 58). Dans d'autres cas, il s'agit de s'approprier les codes culturels issus de la presse magazine grand public et de les détourner : la revue File (fig. 6), anagramme de Life, éditée par le collectif canadien General Idea entre 1972 et 1989 et diffusée dans les kiosques à journaux, reprend le format, la typographie et le logo de l'hebdomadaire américain mais lui donne un contenu thématique différent ( "Glamour », " Punk ») : " alternative à la presse alternative ", cette revue pratique la stratégie d'infiltration des médias et de la culture de masse (Revues d'artistes..., 2008, p. 82-85). Comme les livres d'artistes, ces revues s'approprient les moyens de diffusion de masse pour en faire un contre-usage et s'opposer à leurs effets de massification. Le recours à la reproduction est à la fois " solution artistique et instrument critique " (MEGLINDELCROIX, 2008, p. 177).

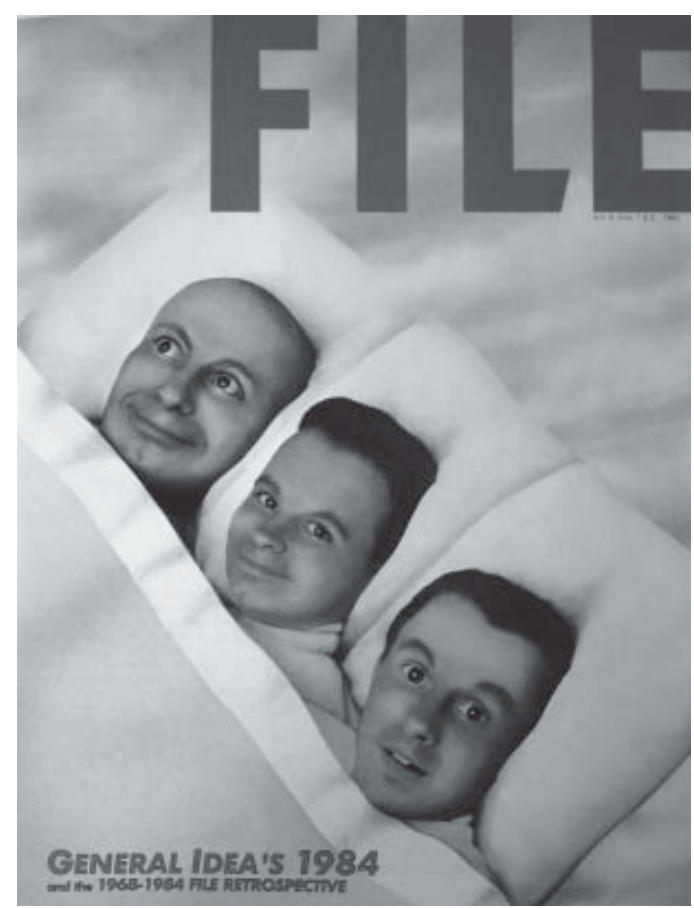

Quel impact ces publications d'artistes ont-elles sur le monde de l'art? Phillpot et Linker, qui dressent un bilan de la pratique du livre d'artiste, s'accordent sur le décalage qui existe entre les aspirations et les revendications des artistes et les effets tangibles qui résultent de leur pratique. En dépit des efforts déployés par les artistes pour faire sortir l'art du circuit traditionnel des galeries et malgré leur prix modique qui devait permettre leur diffusion à un large public, les publications d'artistes sont restées dans le cercle restreint des spécialistes, artistes ou collectionneurs. Linker constate ainsi que le manque de distributeurs a limité la circulation des livres d'artistes, les rendant dépendants des galeries ou des musées qu'ils avaient voulu court-circuiter : l'édition en nombre illimité et la pratique de la gratuité ou du prix de consommation courante n'a pas permis de s'affranchir du réseau marchand, ni même d'échapper à la spéculation du marché de l'art comme en témoigne la transformation des livres d'artistes rares ou épuisés en objets de valeur.

De pratiques marginales qu'elles étaient à leur naissance, les publications d'artistes, autour desquelles se sont peu à peu développés des pôles de diffusion et de recherche, sont devenues un genre artistique à part entière : un paradoxe si l'on rappelle qu'elles visaient à l'origine la destitution de tout genre. Exposées sous vitrine dans les musées, vendues dans les galeries, devenues objets de collections ou de recherches universitaires, elles semblent avoir désormais intégré les institutions alors qu'elles s'étaient affirmées dès leur apparition comme une alternative à cellesci. Cette situation n'implique pas de facto une institutionnalisation, pas plus d'ailleurs que la diffusion élargie ne signifie qu'elles n'auraient su éviter les écueils d'un art de masse. Bien que les réseaux de diffusion en marge du commerce (associations, petites librairies et galeries engagées, travaux universitaire, etc.) se multiplient, ces pratiques de l'art que sont les livres et les revues d'artistes restent encore mal connues du grand public : on peut espérer que le dynamisme des recherches conduites dans ce domaine contribue à la diffusion des " œuvres " d'un genre nouveau.
6. File, revue édité par General Idea, 6/1-2, 1984. 
1. Le Centre national de l'édition et de l'art imprimé (CNEAI), situé sur l'île de Chatou, organise des expositions et produit des œuvres multiples ; ses collections d'éphéméras et de livres d'artistes sont consultables en ligne (www.cneai.com). Le Centre des livres d'artistes (http://cdla.info/fr), basé depuis 1994 dans le Limousin à Saint-Yrieix-la-Perche, et le Cabinet du livre d'artiste de Rennes (www.sites.univ-rennes2.fr/ arts-pratiques-poetiques/incertain-sens/historique_cla. htm) proposent une présentation en ligne de leurs fonds respectifs. Voir également le site www.so-multiples.com.

2. Harold Rosenberg, La Dé-définition de l'art, Paris, 1992 [éd. orig. : The De-Definition of Art, Chicago, 1983].

3. À ce sujet, voir Stephen Bury, "Artists' Multiples, Artists Multiplied ", dans Multiplication, Londres, 2001, exposition itinérante initiée par le British Council à Londres à partir de novembre 2001 (disponible sur www.so-multiples.com/pdf/article_stephenbury_2.pdf), et Anne Mœglin-Delcroix, Esthétique du livre d'artiste (1960-1980), Paris, 1997, p. 121-124 (on annonce pour l'automne 2010 une nouvelle édition de cet ouvrage, épuisé depuis plusieurs années, en coédition par la Bibliothèque nationale de France et Le mot et le reste).

4. Un site interactif présente l'exposition, les textes de présentation de chaque thématique du catalogue ainsi qu'une grande partie des œuvres : www.moma.org/interactives/exhibitions/2006/eyeoneurope/flash.html.

5. Un colloque international, "Le livre d'artiste : quel projet pour l'art ?", est organisé avec le laboratoire "L'œuvre et l'image " de l'université Rennes 2 en collaboration avec l'université de Paris I - Panthéon-Sorbonne, les 18, 19 et 20 mars 2010 à Rennes; il se propose d'interroger le (ou les) projet(s) de l'art implicite(s) au choix fait par les artistes, depuis bientôt cinquante ans, de produire tout ou une partie de leur ouvre sous forme de livres, de revues, d'inserts ou d'autres imprimés. Il réunira des universitaires, des collectionneurs et des artistes.

6. Voir le site de la maison d'édition dont elle est cofondatrice : http://editionsprovisoires.free.fr. Il s'agit d'éditions non limitées et non numérotées, dont certaines sont proposées en version PDF et imprimables.

7. Møglin-Delcroix, 1997, cité n. 3.

8. Kate Linker, "The Artist's Book as an Alternative Space ", dans Studio international, 990, 1980, p. 77-79 (traduit dans BROGOWSKI, MEgGLIN-DELCROIX, 2008, p. 13-17).

9. A Century of Artists'Books, Riva Castleman éd., (cat. expo., New York, Museum of Modern Art, 1994-1995), New York, 1995.

10. Mentionnons les écrits de référence de Phillpot, qui a dirigé la bibliothèque du MoMA de 1977 à 1994 après avoir été responsable de la collection de livres d'artiste ; voir en particulier Clive Phillpot "Books, Books Objects, Bookworks, Artists' Books ", dans Artforum, 20/9, mai 1982, p. 77-79, et, plus récemment, In Numbers. Serial Publications By Artists Since 1955, Andrew Roth et al. éd., (cat. expo., New York, X Initiative, 2009-2010), Zurich/ New York, 2010.
11. Clive Phillpot, texte paru en introduction au catalogue de vente de la librairie Printed Matter Books by Artists, Spring Catalogue, New York, 1990 (traduit dans BROGOWSKI, MEGLIN-DELCROIX, 2008, p. 19-20).

12. Maurizio Nannucci, Art as Social Environnment, Lugo, Exit/Amsterdam, Others Books and So, 1978, 250 exemplaires. Pour plus d'informations sur ses éditions et multiples, voir www.maurizionannucci.it.

13. Maurizio Nannucci, "Art in Bookform/Book in Artform ", publié en anglais et imprimé en capitales annonçant l'exposition d'une sélection de livres d'artistes présentée par Zona Archives ACRL/WESS à Florence, 4-8 avril 1988 (traduit dans BROGOWSKI, MEgLINDELCROIX, 2008, p. 21).

14. Linker, (1980) 2008, cité n. 8.

15. La revue ayant une périodicité fluctuante, un blog (http://revueoxo.blogspot.com) permet de transmettre des informations aux abonnés.

Laurence Corbel, Université Paris I - Panthéon-Sorbonne laurence.corbel@gmail.com 